\title{
Burkholderia Cepacia in Patients with Diabetes Mellitus - An unusal Presentation
}

\author{
Neeti Bhat, ${ }^{1}$ Alina Karna, ${ }^{2}$ Sameer Dutta ${ }^{3}$ \\ ${ }^{1,2}$ Madan Bhandari Academy of Health Sciences, Hetauda \\ ${ }^{3}$ Sukraraj Tropical and Infectious Disease Hospital, Teku, Kathmandu, Nepal. \\ Correspondence: \\ Dr. Alina Karna. Email: alinakarna05@gmail.com
}

\begin{abstract}
Burkholderia Cepacia is an aerobic gram-negative bacillus, rarely affects immunocompetent individuals, usually found in various aquatic environments. We present a case of a 45-year-old male with a known case of diabetes for five years. He presented with high-grade fever, dysuria, and abdominal pain. Routine tests, ultrasonography along together with high-resolution computed tomography was done. High Resolution Computed Tomography showed subtle ground-glass opacity of the superior segment of lingulae of the left lobe of the lungs. Real time polymerase chain reaction was advised to rule out Covid-19 infection, which was negative. Burkholderia Cepacia was identified in CystineLactose-Electrolyte-Deficient Agar culture (CLED).
\end{abstract}

Keywords: Burkholderia Cepacia; Diabetes Mellitus;Urinary tract infection.

\section{INTRODUCTION}

Walter Burkholder first identified Burkholderia cepacia (B. cepacia), formerly known as pseudomonas cepacia in 1950 in roots of onion bulb and is rare organism..$^{1,2}$ B. cepacia are catalase-negative, non-lactose fermenting aerobic, motile, Gram-negative bacilli. ${ }^{2}$ These ubiquitous organisms are found in soil and aquatic environments. ${ }^{3,4} \quad$ B. cepacia primarily affects immunosuppressed patients or with preexisting respiratory epithelial damage, e.g., cystic fibrosis. These organisms are usually immune to multiple antibiotics, and it's known to contaminate intravenous fluids, bronchoscopes, and urinary catheters. ${ }^{4}$ B. cepacia contamination in mouthwash was seen in 2005, which was notified to the Centers for Disease Control. ${ }^{3,4}$

\section{CASE REPORT}

A 42-year-old male who had a fever, dysuria, abdominal fever, and chills for two days presented to the emergency room. His chief complaint also included a mild cough for one day. His past anamnesis includes type 2 diabetes mellitus (DM). The vital signs recorded were temperature $102.3^{\circ} \mathrm{F}$, a heart rate of 130 , respiratory rate of $15, \mathrm{SpO}_{2}$ of $99 \%$, and blood pressure of 130/90 mmHg.

His neurological, respiratory, and cardiovascular exams were all normal. On per rectal examination, the prostate was enlarged, smooth, and non-tender. Blood was sent for complete blood count, fasting and postprandial glucose, Hb1ac, Serum uric acid. A radiological test such as Chest X-ray was HRCT was advised to rule out Covid-19 infection. Rt-PCR was done and was found to be Covid-19 negative. Urine was sent for urine R/E. Both blood and urine were sent for blood culture and antibiotic sensitivity tests. Initial blood tests revealed findings as in Table 1.

Citation: Bhat N, Karna A, Dutta S. Burkholderia Cepacia in patients with diabetes mellitus - an unusual presentation. Nepal J Health Sci. 2021 Jan-Jun;1(1): 64-68 
Routine urine examination showed plenty of pus cells, glucosuria, hematuria, and albuminuria. The uric acid and renal function test (table 2) was within the normal limit. Random blood glucose was $468.8 \mathrm{mg} /$ $\mathrm{dl}$ and Hb1ac was 9.2\%. Chest X-ray (Figure 1A) and HRCT (Figure 1B) of the chest showed subtle groundglass opacity in the superior segment of the lingular. Few pre-tracheal, bilateral paratracheal, carinal, and subcarinal lymph nodes were enlarged, with the largest up to $15 \times 12 \mathrm{~mm}$. However, real time polymerase chain reaction was negative for Covid-19 infection. Ultrasonography of abdomen as shown in figure 3 showed mild dilation of the pelvicalyceal system of left and right kidney, fatty liver (Grade 1). The prostate was enlarged with intravesical prostatic protrusion of five $\mathrm{mm}$ and significant post-void residual urine of $315 \mathrm{ml}$. Intravenous fluids and an antipyretic was started. His urine cultures on cystine-lactose-electrolyte-deficient agar (CLED agar) revealed isolation of $B$. cepacia organism (>105 $\mathrm{CFU} / \mathrm{ml}$ ) on day three as shown in figure 2A. Evaluation of the Antibiotic sensitivity test by Kirby-Bauer disk diffusion showed resistance to gentamicin, nitrofurantoin, and doxycycline as shown in figure 2B. Blood culture was also sent, however, negative. He was treated with injection amikacin and tazobactam. Insulin was started to control the blood sugar.

His chills subsided; however next day of admission, he had a fever of $103.2^{\circ} \mathrm{F}$. His fever subsided on the fourth day. The patient was discharged on seventh day from the hospital. After this episode, he followed up in the clinic after two days. He presented asymptomatic, and his leukocyte count was within normal limits, and urine RE was also normal with only 1-2 pus cells.

Table 1: Complete Blood Count value

\begin{tabular}{l|l|l|}
\multicolumn{1}{c|}{ Tests } & \multicolumn{1}{c|}{ Results } & \multicolumn{1}{c|}{ Reference range } \\
\hline $\begin{array}{l}\text { White blood cells } \\
\text { Red blood cells }\end{array}$ & 21.13 & $4-11(103 / \mathrm{mm} 3)$ \\
\hline Hemoglobin & 3.55 & $4.5-5.5(106 / \mathrm{mm})$ \\
& 9.6 & $\begin{array}{l}\text { Men: } 12-13(\mathrm{~g} / \mathrm{dL}) \\
\text { Women: } 11-13(\mathrm{~g} / \mathrm{dL})\end{array}$ \\
\hline Hematocrit & 28.4 & $\begin{array}{l}\text { Men: } 41-50 \% \\
\text { Women: } 36-48 \%\end{array}$ \\
\hline MCV & 80.0 & $80-100 \mathrm{fl}$ \\
\hline MCH & 27.0 & $27.5-33.2 \mathrm{pg}$ \\
\hline MCHC & 33.8 & $33.4-35.5 \mathrm{~g} / \mathrm{dl}$ \\
\hline RDW $(\%)$ & 12.9 & $11.6-14.6 \%$ \\
\hline Platelet & 1.71 & $1.5-4.5(105 / \mathrm{mm} 3)$
\end{tabular}

Table 2: Renal function test(RFT) value and uric acid

\begin{tabular}{l|l|l|}
\multicolumn{1}{c|}{ Tests } & \multicolumn{1}{c|}{ Results } & \multicolumn{1}{c}{ Reference range } \\
\hline Serum urea & 30.2 & $15-45 \mathrm{mg} / \mathrm{dl}$ \\
\hline Serum Creatinine & 1.1 & $0.4-1.4 \mathrm{mg} / \mathrm{dl}$ \\
\hline Sodium & 131.5 & $135-155 \mathrm{mmol} / \mathrm{l}$ \\
\hline Potassium & 3.8 & $3.5-5.5 \mathrm{mmol} / \mathrm{l}$ \\
\hline Ionized calcium & 1.11 & $1.05-1.35 \mathrm{mmol} / \mathrm{l}$ \\
\hline Serum uric acid & 5.2 & $2-7 \mathrm{mg} / \mathrm{dl}$
\end{tabular}




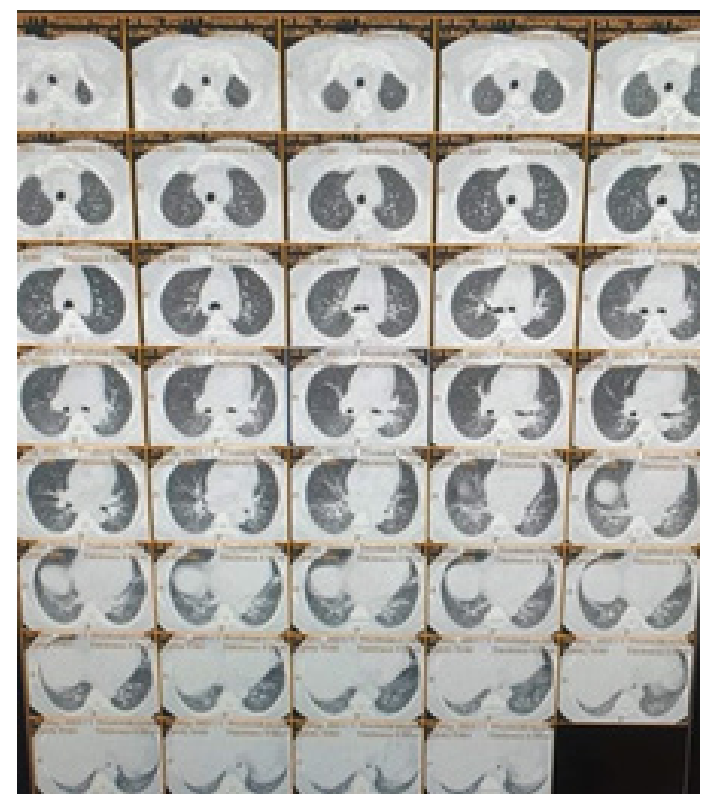

1.A

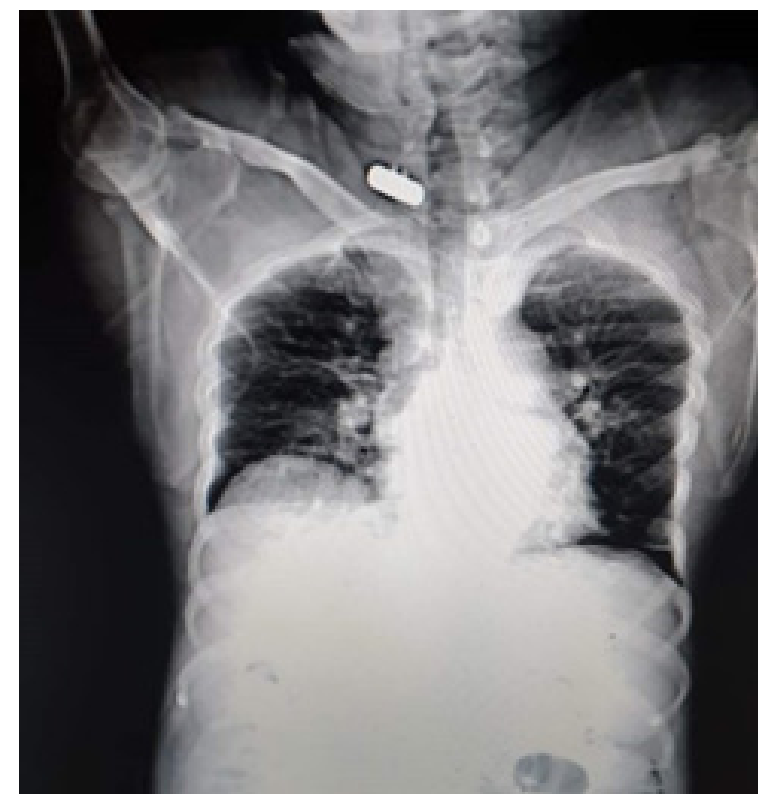

1.B

Figure 1.A and 1.B: HRCT (right) and Chest X-ray (left) showing subtle ground glass opacity in superior segment of lingular lobe.

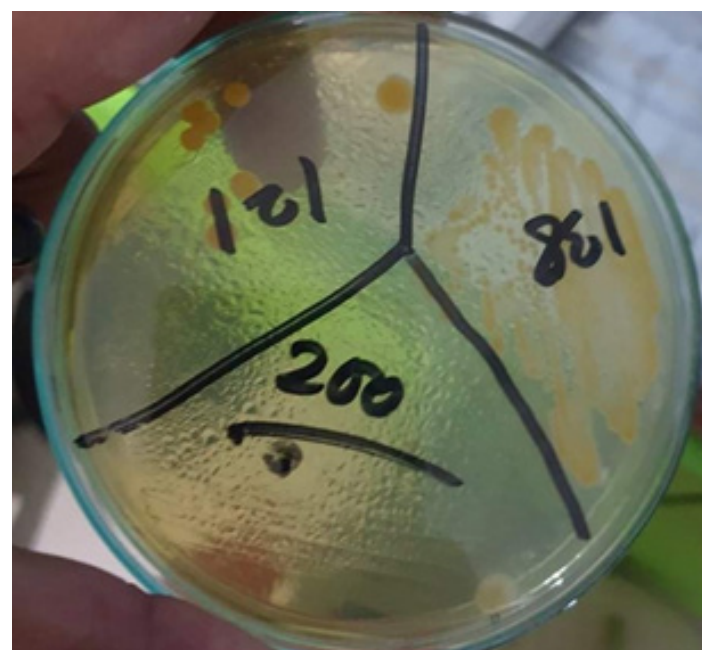

2.A

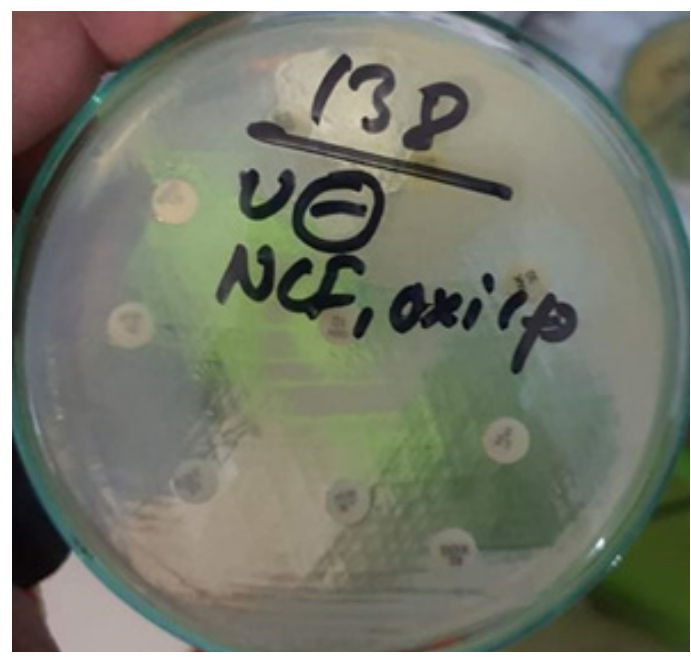

2.B

Figure 2.A and 2.B: Urine culture in CLED agar showing growth of Burkholderia cepacia (right) and antibiotic sensitivity test (left) in Kirby-Bauer disk diffusion showed resistance to gentamicin, nitrofurantoin and doxycycline.
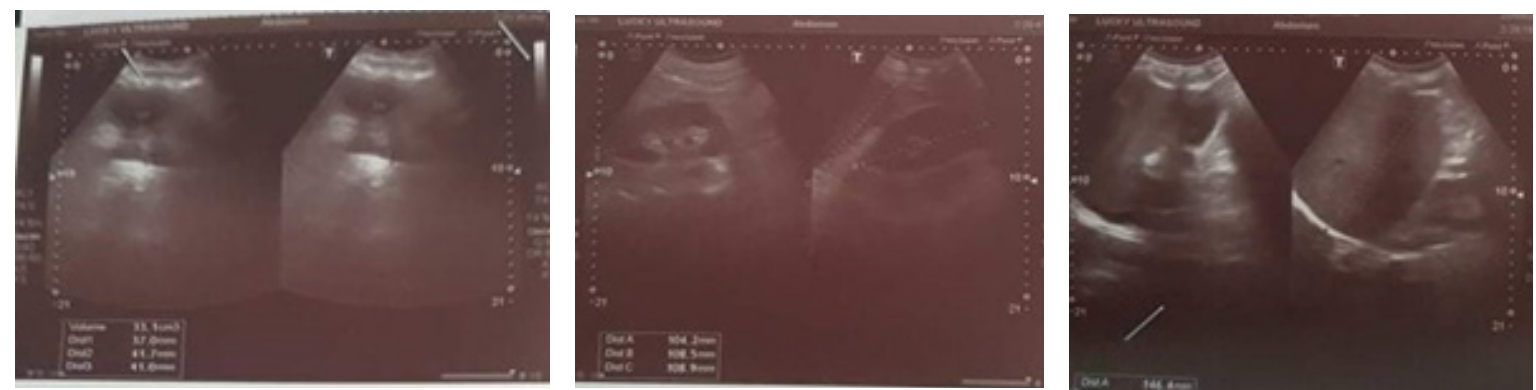

Figure 3: USG abdomen showing mild dilation of the pelvicalyceal system of bilateral kidney, fatty liver (Grade 1) and mild prostatomegaly. 


\section{DISCUSSION}

Burkholderia cepacia (BC) is an emerging pathogen that causes life-threatening infections. There have been several reports of BC outbreaks due to contamination of medications and equipment. Farmers commonly use $\mathrm{BC}$ for crop growth due to its fungicidal and pesticide metabolizing activity and its antagonistic action against several plant pathogens. The use in farms may lead to water contamination. B. cepacia infection is usually common in patients with respiratory epithelial dysfunction, immunosuppressed individual and a few catheter-induced bacteremia in hemodialysis patient. ${ }^{5}$ There were many reports of $B$. Cepacia presenting as pneumonia in cystic fibrosis patients and some immunocompromised individuals. This case report is interesting because the patient had diabetes, wasn't dialysis-dependent and presented with urinary tract infection. The urine culture revealed isolation of $B$. cepacia while blood culture was negative. The underlying reason for B. cepacia urinary tract infection could be urine tract obstruction due to prostate hypertrophy. Clinical manifestations are varied from asymptomatic colonization to sepsis, necrotizing pneumonia and infrequently urinary tract infection. The treatment guidelines had not been established yet. These organisms are often sensitive to trimethoprim-sulfamethoxazole, meropenem, amikacin, tazobactam and ceftazidime. ${ }^{6}$

Antibiotic therapy, often in combination when intravenously administered, has been successful. Burkholderia species are often immune to multiple drugs like our case, and treatment is challenging. The genetic resistance mechanism of Burkholderia spp to anti-microbial is histidine kinase ( $\mathrm{mrgS}$ ) and its regulatory component (mrgR), modified lipopolysaccharides, decreased outer membrane permeability of Burkholderia complex and efflux pump. Burkholderia complex is naturally resistant to many anti-microbial agents including carboxypenicillins, polymyxin, and often, aminoglycosides due to the efflux pump activity. ${ }^{7}$ A thorough literature review suggested that our overall understanding of resistance in B. cepacia is still rather rudimentary. Zeeshan et al reported recurrent $B$. cepacia infection in post-renal transplant patients in Sindh Institute of Urology and Transplantation, Karachi, which is a rare finding. ${ }^{8}$ Nimiri et al. also reported four urinary tract infections caused by $B$. Cepacia complex in patients with no underlying risk factor. The author asserted that highly anti-microbial resistance organisms such as $B$. cepacia should not be ignored when patients present with UTI, which may lead to treatment failure. ${ }^{9}$

\section{SUMMARY}

The occurrence of B. cepacia infection in people with diabetes with noted urine outflow tract obstruction presenting as urinary tract infection needs to be studied.

$\begin{array}{ll}\text { Abbreviation : } & \text { CFU: Colony forming unit } \\ & \text { - } \\ & \text { } \text { trolyte Deficient agar } \\ & \text { HRCT: High Resolution } \\ & \text { Computed Tomography } \\ & \text { RE: Routine Examination } \\ & \text { - RFT: Renal Function Test } \\ & \text { UTI: Urinary Tract Infection }\end{array}$

\section{Conflict of interest : None}

\section{REFERENCE}

1. Bayram M, Babalık M, Bakan ND, Döngel I. Community-acquired Burkholderia cepacia pneumonia: a report of two immunocompetent patients. Tuberk Toraks. 2011 Jan 1;59(4):380-3. PMID: 22233309, doi: 10.5578/tt.1159.

2. Zuckerman JB, Seder DB. Infection control practice in cystic fibrosis centers. Clin Chest Med.2007 Jun 1;28(2):381-404. PMID: 17467555, doi: 10.1016/j.ccm.2007.02.010.

3. Oyesina O, Anthony WC. Burkholderia Cepacia: A case report. J Med Cases. 2014 Jan 3;5(1):9-10. doi: https://doi.org/10.4021/jmc1425e

4. Long. Principles and Practice of Pediatric Infectious Diseases Revised reprint, 3rd ed. Churchill 
1. Livingstone, An Imprint of Elsevier. 2009.

2. Mandell. Mandell, Douglas, and Bennett's Principles and Practice of Infectious Diseases, 7 th ed. Churchill Livingstone, An Imprint of Elsevier. 2009. doi: 10.1016/S1473-3099(10)70089-X

3. Zhou J, Chen Y, Tabibi S, Alba L, Garber E, Saiman L. Antimicrobial susceptibility and synergy studies of Burkholderia cepacia complex isolated from patients with cystic fibrosis. Antimicrob Agents Chemother. 2007;51(3):1085-1088. PMID: 17158942 doi:10.1128/AAC.00954-06.

4. Sfeir MM. Burkholderia cepacia complex infections: more complex than the bacterium name suggest. Jou Inf. 2018 Sep 1;77(3):166-70. Doi: https://doi.org/10.1016 /j.jinf.2018.07.006

5. Zeeshan M, Aziz T, Naqvi F. Recurrent urinary tract infection by Burkholderia cepacia in a live related renal transplant recipient. Jou Pak Med Asso. 2012 May; 62 (5). https://jpma.org.pk/article-details/3406?article_id=3406

6. Nimri L, Sulaiman M, Hani OB. Community-acquired urinary tract infections caused by Burkholderia cepacia complex in patients with no underlying risk factor. JMM case reports. 2017 Jan;4(1). doi: 10.1099/jmmcr.0.005081

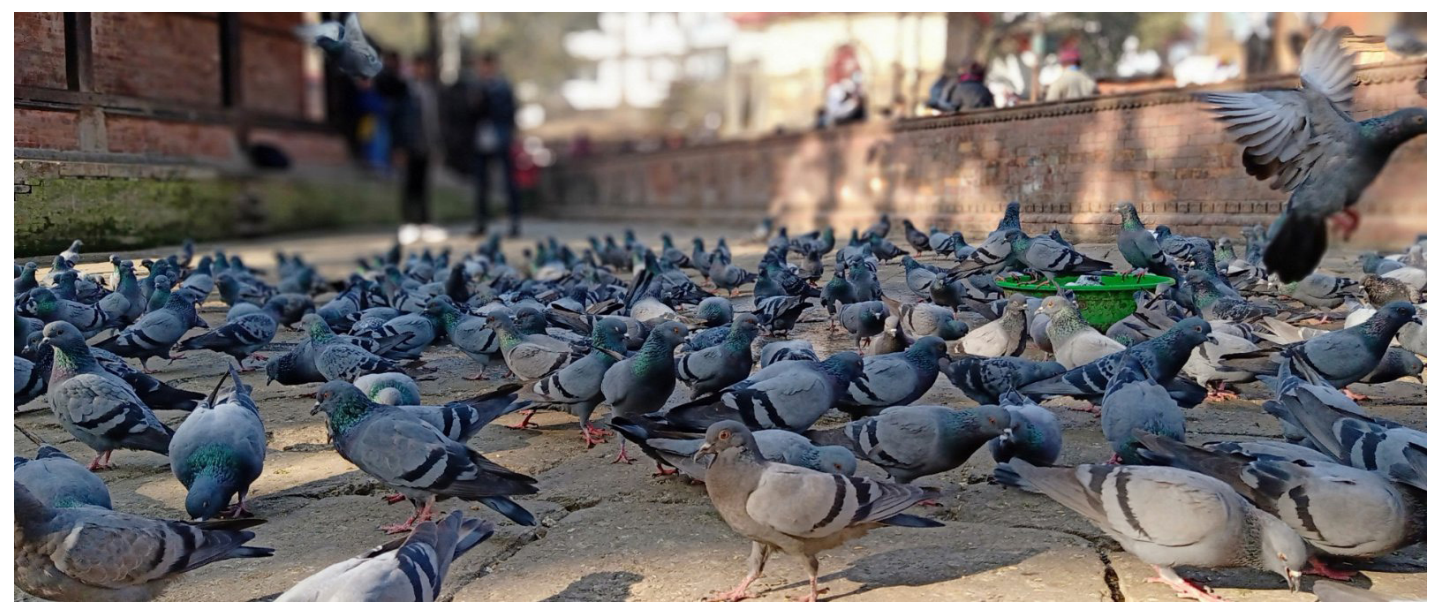

Symbol of Love and Peace

-Sailendra Chaudhary, M.Pharma 